\title{
MOLA HIDATIDIFORME BENIGNA
}

\author{
Dr. Alberto Zabaleta Lombana* \\ Dr. Jorge Milanés Pernett**
}

Poco es el interés que la literatura médica nacional ha prestado a la llamada Mola Hidatidiforme Benigna; no obstante que su ocurrencia en nuestro medio es aparentemente alta, justificando una más despierta atención.

Las estadísticas de diferentes países señalan una mola por cada 2.000 a 2.500 gestaciones. En el lapso de 8 años, hasta 1962, Acosta Bendeck en Barranquilla (1) encontró una alarmante incidencia de 168 casos sobre 52.358 embarazos; una por cada 311. Valencia Hurtado y colaboradores (2) Ilamaron la atención en el $V$ Congreso Colombiano de Obstetricia y Ginecología de Medellín, 1963, acerca de la gran frecuencia de esta entidad en el Departamento de Caldas. En un período de seis y medio años, hasta el primer trimestre de 1963 ocurrieron 91 mola entre 14.163 embarazos promediando una por cada 155.

Tal índice equipara esa sección del país en relación con esta misma patología con las Filipinas y el sur oriente asiático, en donde representa

* Profesor Asistente del Departamento de Obstetricia y Ginecología. Facultad de Medicina. Cartagena.

** Jefe del Departamento de Obstetricia y Ginecología. Facultad de Medicina. Universidad de Cartagena. uno de 145 embarazos. Es bueno destacar que los autores caldenses computan en su trabajo la micro lesión edematosa, hidrópica, de la vellosidad corial encontrada al estudio anatomo-patológico de los especímenes de aborto. No compartimos esta amplitud de concepto, por cuanto el edema de la vellosidad puede no ser en estos casos más que un proceso a título degenerativo, como resultado del trastorno de su circulación, particularmente cuando muere el embrión o feto. Por ello, se observa en ocasiones en el embarazo ectópico, muerte fetal in útero, toxemia y en la placenta hidrópica de la eritroblastosis. Así mismo, se ha señalado esta micro-lesión en el $20 \%$ de los casos de aborto espontáneo; junto a zonas placentarias bien conservadas - afectas de degeneración hialina, fibrosa o vellosidades necróticas. Duarte Contreras y col. (3) encontraron esta degeneración hidrópica en el $18,4 \%$ en 103 casos de aborto; considerándola posiblemente ligada a cambios vasculares responsables de una mala irrigación. Isaza Mejía (4), en el $24,8 \%$ sobre 137 preparaciones histológicas por aborto junto a otras alteraciones vellosas como ausencia de vasos, sincitio con vacuolas, ca pa de Langhans atrófica, etc.

Ahora bien, para mayor abundamiento, debemos recordar que la mi- 
crolesión hidrópica de la vellosidad en los casos de aborto, rara vez se acompaña de la hiperplasia trofoblástica, característica histológica de la mayor importancia en la mola, mucho más que su edematosa morfología. La mola es considerada como la expresión de un producto abortivo de la concepción en el que las vellosidades se hacen vesiculosas con un grado mayor o menor de reacción trofoblástica; en tanto que el embrión, salvo contadas excepciones, no existe. Por ello, ha sido catalogada en el grupo I de Moll, de la clasificación Carnegie para los huevos patológicos (5).

\section{MATERIAL Y METODO}

El presente trabajo lo constituye la revisión de 96 casos de mola hidatidiforme, con diagnóstico clínico hecho evidente por la presencia de los característicos racimos de vellosidades vesiculosas. La contabilidad se hizo parte en el archivo del Departamento de Anatomía Patológica en número de 28; 62 del examen de historias clínicas de la Maternidad "Rafael Calvo C." y las restantes 6 en la estadística de algunas instituciones privadas de la ciudad.

Los datos obtenidos han sido agrupados en cuadros que comprenden la paridad, embarazo al que corresponde la mola, frecuencia de la misma, relación con el número de embarazos, partos y abortos por grupos de edades; duración de la gestación molar y condiciones generales del paciente de acuerdo con algunos porcentajes hematológicos. En otros cuadros comparamos algunos aspectos del grupo molar con una muestra de pacientes que no padecieron dicha enfermedad.

En el cuadro número 1 apuntamos el estado de la vellosidad corial señalado por el patólogo en 180 casos de aborto revisados por nosotros; para destacar que la microlesión edematosa se encuentra asociada, como una más, a otras manifestaciones de sufrimiento y muerte vellosa.

\section{CUADRO N: 1}

\section{ALTERACIONES VELLOSAS EN EL ABORTO}

\begin{tabular}{lcc}
\hline Estados de la Vellosidad & Veces & $\%$ \\
\hline Normales & 4 & 38.8 \\
Más o menos bien preservadas & 70 & 10. \\
Degeneración de distintos grados & 18 & 6. \\
Reacción Trofoblástica intensa-moderada & 11 & 17.7 \\
Hialinización & 32 & 8.9 \\
Edematización & 16 & 23.6 \\
Necrosis & 42 & 14.8 \\
Infiltración por polinucleares, linfocitos, etc. & 26 & 2 \\
Hemorrágicas & & \\
\hline
\end{tabular}

Observamos en escaso porcentaje, 8.9, la edematización vellosa, probablemente porque no fué buscada sistemáticamente. Por lo demás, las distintas características anotadas pueden hallarse conjuntamente presentes o bien ausentes una $u$ otra en diferentes cortes de un mismo espécimen. 
CUADRO N: 2

CASOS HISTORIADOS POR AÑO

\begin{tabular}{cc}
\hline Años & Casos \\
\hline 1954 & \\
1955 & 8 \\
1956 & 3 \\
1957 & 2 \\
1958 & 3 \\
1959 & 4 \\
1960 & 6 \\
1961 & 5 \\
1962 & 7 \\
1963 & 9 \\
1964 & 19 \\
1965 & 7 \\
\hline
\end{tabular}

La catalogación por año se hace por simple método; ya que parcialmente y en conjunto hasta 1964 no representa las cifras exactas de su incidencia que resulta ser realmente más alta. Esta misma razón nos impide establecer relación con el número de embarazos o abortos.

En el año de 1965 solo comprende los primeros 9 meses hasta el 30 de Septiembre. En este lapso, en la unidad obstétrica universitaria, han sido atendidas 19 molas, 845 abortos y 3.893 partos; lo que representa una mola por 44,5 abortos y una por 250 gestaciones.

\section{CUADRO NN 3}

FRECUENCIA DE LA MOLA POR GRUPOS DE EDADES - 92 CASOS

\begin{tabular}{lccc}
\hline Grupos de Edades & $\mathbf{N}^{0}$ de Casos & Porcentajes \\
\hline 13 a 19 años & 19 & 20.65 \\
20 a 29 años & 38 & 41.30 \\
30 a 39 años & 18 & 19.56 \\
41 a 49 años & 14 & 15.21 \\
$50-55-58$ años & 3 & 3.26 \\
\hline
\end{tabular}

En realidad, se puede considerar alta la incidencia de mola en todos los grupos de edades, particularmente entre los 20 y 30 años. Como se ve en el Cuadro № 3, las gestaciones en el curso de la adolescencia también están gravadas con un gran porcentaje de mola; constituyendo el $20.65 \%$ en esta estadística.

Las edades extremas fueron 13 años en una primigrávida y 58 en una gran multípara. Sería esta última la paciente más vieja conocida abortada de mola. Sin embargo, el relato de su edad no tiene el respaldo de certificación legal alguna. Su historia fué conocida por el protocolo de patología número 13856/61. En la Obstetricia de Williams, Eastman anota embarazos molares a las edades de 12 y 55 años (6). Destacamos las edades de otras pacientes: $14,15,49,50$ y 55 años.

El hecho que la mayor incidencia tenga lugar en el tercer decenio de la vida $(41,30 \%)$, no se debe a que corresponda a la zona de mayor actividad genital de la mujer. Examinando los índices de gravidez de distintos grupos de edades, como reflejo de la actividad sexual obstétrica, encontramos que son sensiblemente iguales en todos los niveles; por lo que ningún grupo tendría más oportunidad que otros de dar lugar a un embarazo molar u otra cualquiera patología. (Ver Cuadro № 6).

Buscando verificar una eventual relación entre los índices de gravidez, paridad y aborto con la mola, investigamos una muestra de 1.892 pacientes que no habían tenido embarazo molar, cuyos datos comparamos con similares apartes del grupo molar, detalles que reseñamos en los Cuadros Nos. 4 y 5 . En ambos, agrupamos a las pacientes en iguales escalas de edades. Los índices los obtenemos dividiendo la cifra de gestaciones, partos y abortos de cada grupo por el correspondiente número de casos. 


\section{CUADRO NN 4}

\subsection{PACIENTES SIN EMBARAZO MOLAR}

\begin{tabular}{rrrrrrrrr}
\hline & \multicolumn{9}{c}{ Número de : } & \multicolumn{3}{c}{ Indices de : } \\
Grupos de edades & Casos & \multicolumn{1}{c}{ G. } & P. & A. & G. & P. & A. \\
\hline Menos de 20 años & 326 & 547 & 485 & 62 & 1.67 & 1.48 & 0.19 \\
20 & a 29 años & 996 & 3.843 & 3.336 & 507 & 3.86 & 3.36 & 0.50 \\
30 & a 39 años & 503 & 3.614 & 3.162 & 452 & 7.18 & 6.29 & 0.98 \\
40 & a 49 años & 67 & 670 & 571 & 99 & 10 & 8.53 & 1.47 \\
TOTAL & 1.892 & 8.674 & 7.554 & 1.120 & 4.58 & 3.99 & 0.59 \\
\hline
\end{tabular}

En resumen, 1.892 pacientes sin embarazo molar tuvieron 8.674 gestaciones que terminaron por 7.554 partos y 1.120 abortos, con índices de gravidez, paridad y aborto de $4.58 ; 3.99 ; 0.59$ respectivamente.

\section{CUADRO N: 5}

\section{PACIENTES CON EMBARAZO MOLAR: 61}

\begin{tabular}{|c|c|c|c|c|c|c|c|c|c|}
\hline \multirow[b]{2}{*}{ Grupos } & \multirow{2}{*}{\multicolumn{2}{|c|}{ de edades }} & \multicolumn{4}{|c|}{ Número de: } & \multicolumn{3}{|c|}{ Indices de: } \\
\hline & & & Casos & G. & P. & A. & G. & P. & A. \\
\hline Menos & de & 20 años & 15 & 25 & 5 & 20 & 1.66 & 0.33 & 1.33 \\
\hline 20 & $a$ & 29 años & 26 & 102 & 63 & 39 & 3.92 & 2.42 & 1.50 \\
\hline 30 & a & 39 años & 7 & 59 & 48 & 11 & 8.42 & 6.85 & 1.57 \\
\hline 40 & $\mathrm{a}$ & 49 años & 13 & 167 & 115 & 52 & 12.84 & 8.84 & 4. \\
\hline TOTAL & & & 61 & 353 & 231 & 122 & 5.78 & 3.78 & 2 \\
\hline
\end{tabular}

El Cuadro № 5 -pacientes con embarazo molar- resume 61 casos cuyos correspondientes datos aparecieron en las historias clínicas. En la cuenta de los abortos se incluyó como tal la mola. No se reseñó un caso de embarazo a término con placenta succenturiada molar.

Se observa en los anteriores Cuadros 4 y 5 que los índices de gravidez son sensiblemente iguales por grupos de edades, excediendo un tanto en los totales a favor de las molas. El índice de paridad resulta indiferente por grupos de edades; $y$ más o menos iguales en los totales. Por el contrario, el índice de aborto se eleva notablemente en las pacientes con embarazo molar por escala de edades y en el total; siendo su relación de 3.4 a 1 en beneficio de estas últimas. Este hecho parecería señalar la presencia de un número mayor de óvulos genéticamente patológicos en estas pacientes abortadoras, similar a lo que ocurre en los mamíferos que tienen ordinariamente gran número de crías (7). 
CUADRO N: 6

COMPARACION DE INDICES

\begin{tabular}{|c|c|c|c|c|c|c|}
\hline \multirow{2}{*}{$\begin{array}{l}\text { № } \\
\text { G. }\end{array}$} & \multicolumn{2}{|c|}{$\begin{array}{l}\text { Molares: } 1.892 \text { casos } \\
\text { Indices de: }\end{array}$} & \multirow[t]{2}{*}{ Grupos de edades } & \multicolumn{3}{|c|}{$\begin{array}{c}\text { Embarazo Molar: } 61 \text { casos } \\
\text { Indices de: }\end{array}$} \\
\hline & P. & A. & & G. & P. & A. \\
\hline 1.67 & 1.48 & 0.19 & Menos de 20 años & 1.66 & 0.33 & 1.33 \\
\hline 3.86 & 3.36 & 0.50 & 20 a 29 años & 3.92 & 2.42 & 1.50 \\
\hline 7.18 & 6.29 & 0.89 & 30 a 39 años & 8.42 & 6.85 & 1.57 \\
\hline 10. & 8.53 & 1.47 & 40 a 49 años & 12.84 & 8.84 & 4. \\
\hline 4.58 & 3.99 & 0.59 & TOTALES : & 5.78 & 3.78 & 2 \\
\hline
\end{tabular}

En el Cuadro № 6 destacamos estos detalles comparando los índices de los dos grupos molar y no molar por escala de edades.

CUADRO NN 7

EMBARAZO CORRESPONDIENTE A LA MOLA

\begin{tabular}{crr}
\hline Embarazo $N^{0}$ & Casos & $\%$ \\
\hline 1 & 15 & 24.6 \\
2 & 6 & 9.8 \\
3 & 10 & 16.3 \\
4 & 2 & 3.3 \\
5 & 3 & 4.9 \\
6 & 3 & 4.9 \\
7 & 1 & - \\
8 & 3 & 4.9 \\
9 & 4 & 6.5 \\
10 & 1 & 18. \\
más 10 & 11 & 3.3 \\
$19-20$ & TOTAL : 61 casos & \\
\hline
\end{tabular}

El Cuadro N: 7 lo constituyen los datos de 61 historias en las cuales se anotaba tal antecedente. Señala el hecho de la mayor frecuencia de la mola en el curso de los tres primeros embarazos en los cuales tuvo lugar el $50,7 \%$ de las mismas. Se destaca la primigravidez, 24,6\%, la tercigravidez, $16,3 \%$ y la multigravidez con más de 10 embarazos, $18 \%$. Hubo dos casos en los cuales las pacientes completaban 19 y 20 gravideces con la gestación molar.

En las primigrávidas, las edades fluctuaron entre los 13-19 años (8 casos), 21-23 ( 6 casos); y una de 28 años. En el grupo de más de 10

CUADRO NN 8

\section{DURACION DEL EMBARAZO MOLAR}

\begin{tabular}{rrr}
\hline \multicolumn{1}{c}{ Días } & Casos & $\%$ \\
\hline $45-90$ & 19 & 24.4 \\
$91-150$ & 44 & 56.4 \\
$150-180$ & 10 & 12.8 \\
188 & 1 & \\
$203-215$ & 2 & \\
$275-280$ & 2 & \\
& TOTAL: 78 casos \\
\hline
\end{tabular}

embarazos las dos menores tenían 38 y 35 años respectivamente; y las restantes 9 variaban entre 41 y 46 años.

Integramos el Cuadro Ni 8 con los datos de 78 pacientes, contabilizando el tiempo transcurrido entre el primer día de la última menstruación y la fecha de expulsión del producto molar.

A este respecto, parecería como si la vellosidad tuviera un límite determinado de actividad al final del cual es abortada. Generalmente el máximo de duración alcanza 150-180 días $(12,8 \%)$ y el término mediano va entre los 90 y 150 días $(56,4 \%)$. Encontramos en nuestra observación tres períodos de duración del embarazo molar, en base de los cuales de- 
nominamos el aborto de la siguiente manera:

A) Aborto Precoz Molar: 19 casos. La gestación alcanza el mínimo de duración, variable entre los 45 y 90 días. Tres pacientes abortaron a los $45-48$ y 51 días respectivamente. Total: $24,4 \%$.

B) Aborto Prematuro Molar: 44 casos. La expulsión de la mola ocurre entre los 91 y 150 días. 56,4\%.

C) Aborto Molar Tardío: La gestación molar alcanza entre 151 y 180 días. Hubo un total de 10 casos con porcentaje de 12.8. Por su interés relataremos más adelante los dos casos que duraron 275 y 280 días, respectivamente -embarazos a término-. La mola que alcanzó 215 días correspondió a la paciente de 58 años.

No hay relación entre la edad y número de embarazos con la dura- ción de la gestación molar. Cuatro pacientes mayores de 40 años abortaron entre los 151 y 180 días; en tanto que dos más lo hacían a los 51 y 89 días. Todas habían tenido más de 10 embarazos. Las 2 pacientes más jóvenes 13 y 14 años, primigrávidas, abortaron a los 114 y 122 días respectivamente. Otras de 15, 16 y 17 años con 3-2 y 2 embarazos, abortaron a los 165-137 y 66 días, en su orden.

\section{Condiciones Generales del pacien-} te: En la gran mayoría de los casos, la mola se manifiesta como una entidad anemiante. El estado nutricional anterior, la metrorragia y la demora en tomar una conducta activa, todo contribuye a provocar un déficit de las condiciones generales del paciente que se refleja en sus valores hematológicos. En el Cuadro № 9 hemos agrupado el conteo de hematíes, hemoglobina y hematocrito encontrados en 38 casos.

CUADRO N: 9

ALGUNOS VALORES HEMATOLOGICOS

\begin{tabular}{cccccccccc}
\hline & Hematíes & x millones & \multicolumn{4}{c}{ Hematocrito } & $\%$ & Hemoglobina & (grs.) \\
\hline & $1-2$ & $2-3$ & $3-4$ & 10 & 20 & 30 & $5-8$ & $8-9$ & más \\
& & & & 20 & 30 & 39 & & 10 \\
Casos & 6 & 17 & 8 & 5 & 15 & 12 & 18 & 9 & 4 \\
\hline
\end{tabular}

Se destaca la pobreza de los factores hemáticos de estas pacientes si consideramos que 23 de ellas apenas alcanzan los 3 millones de glóbulos rojos $(60,5 \%) ; 20$ no pasan del $30 \%$ de hematocrito y 27 Ilegan a los 9 gramos de hemoglobina.

Sintomatología: El cuadro sintomatológico de la mola es muy diciente en la generalidad de los casos. Su expulsión frecuentemente ocurre en el curso de los cinco primeros meses, precedidas siempre de un período más o menos largo de metrorragia cuya principal característica es la falta de dolor. Esta condición puede en un momento dado tener gran valor en el diagnóstico diferencial. Queremos llamar la atención hacia esta particularidad de la metrorragia molar a la cual damos toda la importancia que la misma tiene en el tercer trimestre de la gestación 
para el diagnóstico presuntivo de placenta previa. Es preciso, sí, solicitar cuidadosamente este detalle pues el dolor siempre está presente días $u$ horas antes de provocarse el aborto. En la revisión de 60 historias encontramos este tipo de metrorragia intermitente, indolora en 44 $(73.3 \%)$. Creemos que por poca acuciosidad no se insistió en la falta del elemento dolor tiempo antes del momento de la consulta. Algunos casos se acompañaron de vómitos, edema e hipertensión.

Otros detalles para el diagnóstico lo constituyen en ocasiones la disparidad de tamaño del útero en relación a la edad del embarazo, según fecha de última regla y la notable ocupación de los fondos de saco laterales. Nos parece de poco interés diagnóstico la reacción biológica cualitativa, por cuanto no evidencia su positividad más que la presencia de tejido corial activo.

Tratamiento: A todas las pacientes se les practicó legrado uterino con las medidas complementarias necesarias. A algunas se les hizo histerectomía.

Anatomía Patológica: 28 casos pertenecientes a los primeros años reseñados, se conocieron por revisión del archivo de anatomía patológica. De los restantes 68 , solo 32 tienen estos informes; conformando un total de 60 estudios de patología $(62,5 \%)$.

Una característica importante de la mola benigna es la conservación del patrón histológico de la vellosidad normal, no obstante el estado hidrópico de su estroma y la escasez o ausencia de vasos sanguíneos. Generalmente su cubierta epitelial presenta un grado mayor o menor de hiperplasia.
El interés de este examen para los especímenes de mola radica en la posibilidad de conocer la severidad de la reacción trofoblástica, el atipismo de sus elementos y grado de penetración en los tejidos vecinos, para lo cual es de desear una muestra que contenga tejido uterino. Sin embargo, ninguno de estos elementos faculta al patólogo para pronunciarse sobre el porvenir de una paciente con mola y menos calificar definitivamente la aparente benignidad del tejido corial. El examen anatomo-patológico asociado a posteriores titulaciones hormonales gonadotrópicas y una estrecha vigilancia clínica, constituyen el fundamento de un buen control en caso de aborto molar.

Varios. Dos pacientes tuvieron mola embrionada, con feto macerado. Otras dos relataron antecedentes de aborto molar. Cinco molas fueron invasoras (Molas Malignas, Corioadenoma Destruens) tratadas por histerectomías $(5,2 \%)$. Estas ocurrieron en las pacientes con las más bajas cuentas hematológicas, así: hematíes entre 1.280 .000 y 2.370 .000 -hemoglobina, entre 5.3 y 6.5 grs. y hematocrito entre $10 \%$ y $25 \%$-. El tiempo transcurrido entre la expulsión molar y el diagnóstico de malignidad no excedió de 1 mes. En dicho intervalo estas pacientes continuaron en mal estado general y con metrorragias esporádicas que condujeron al diagnóstico y a la histerectomía. No hubo en estos 96 casos de mola ninguna degeneración corioepiteliomatosa.

En 32 historias se hizo constancia del embarazo anterior a la mola. En 26, parto a término; en 6 , aborto. Un caso 10 años entre el aborto y la mola. 
De 66 historias, en 16 se anotaron quistes luteínicos $u$ ovarios poli-quísticos uni o bilaterales $(24.2 \%)$. Generalmente fueron descubiertos en los días siguientes a la expulsión de la mola, con desaparición espontánea. Los más voluminosos se consideraron concomitantes. Tres pacientes fueron intervenidas por el tamaño de los quistes y molestias adicionales causadas por los mismos.

\footnotetext{
HISTORIA : Relatamos suscintamente un caso de mola embrionada y otro de placenta succenturiada en embarazo a término, con cotiledón ectópico molar. El primer caso corresponde a una paciente de 23 años. G. 3 P. 1 A. 1. Primer embarazo termina por aborto $y$ curetaje en 1960. Diez y ocho meses más tarde, parto a término. Ultima regla: junio 5-8/ 62. En marzo $8 / 63$ consulta por disminución de la altura uterina. Se practica inducido y curetaje, extrayéndose placenta con degeneración molar y un feto papiráceo.
}

El segundo caso fué conocido en los archivos de anatomía patológica. Informe 12243/59. Paciente G. 3 P. 1 A. 1 de 33 años. La primera gestación terminó por aborto 10 años antes. Dos años más tarde, parto prematuro con feto muerto. El tercer embarazo, parto a término, laborioso en diciembre 15/59. Feto vivo de 3.800 grs., placenta con peso de 450 grs., de aspecto normal, completa. Revisada la cavidad uterina por hemorragia post-partum, se extrae pequeña masa placentaria constituída por varios cotiledones con degeneración molar parcial. Se practicó correspondiente estudio de patología cuyo número de protocolo hemos anotado.

\section{RESUMEN}

Se presentan las observaciones recogidas en 96 casos de embarazo molar, 60 de los cuales tienen informe de patología. Se analiza el valor de la microlesión edematosa de la vellosidad corial para catalogar los especímenes como mola.
Por el tiempo de duración del embarazo, se propone su división en aborto molar precoz, cuando alcanza hasta los 90 días; prematuro, hasta los 150 días y tardío cuando la mola se expulsa próximo a los 180 días. Encontramos en nuestros casos un mayor índice de aborto en las pacientes con mola; en relación con un grupo de 1.892 mujeres sin gestación molar. Se llama la atención sobre el carácter indoloro de la metrorragia en la sintomatología de la mola; y deficientes condiciones generales manifestadas en los bajos valores hematológicos. $24,2 \%$ de las pacientes presentaron quistes luteínicos $u$ ovarios poliquísticos, uni o bilaterales.

Cinco $(5,2 \%)$ fueron mola maligna, invasora, tratadas mediante la histerectomía. No hubo muerte materna alguna. Ninguna mola degeneró en corioepitelioma.

\section{BIBLIOGRAFIA}

1 ACOSTA BENDECK, A.: Tumores del Trofoblasto. Rev. Col. Obs. Gin. Vol. XV Ni 5 . Sept.-Oct. 1964.

2 VALENCIA HURTADO, F. y col. Mola Hidatidiforme. Rev. Col. Obst. Gin. Vol. XV. N? 4. Jul.-Agost./64.

3 DUARTE CONTRERAS, A. y col. Estudio completo de 235 abortos. Rev. Col. Obst. Gin. Vol. XV. N․ 5. Sep.-Oct. 1964.

4 ISAZA MEJIA, G.: 270 raspados uterinos para el Aborto. Rev. Col. Obs. Gin. Vol. XI. N: 5 Sept.-Oct./60.

5 NOVAK E., R. WOODRUFF, J. D.: Gineco logic and Obstetric Patology. Cap. XXXIII, pág. 551, 5a ed. inglesa. W. B. Saunder, Philadelphia.

6 EASTMAN, N. J. Obstetricia de Williams. 3? Ed. en Español. H. Vela Treviño. Pág. 555. Uteha, México 1960.

7 EASTMAN, N. J. Edición cit. pág. 497. 